Si mul at i on of ampl i tude and phase char act eristics during i nspecti on of printed ci rcuit board by eddy-current testing probe

\begin{tabular}{|l|l|}
\hline 著者 & Kacpr zak D. Yamada Sot oshi , I wahar a Masayoshi \\
\hline 著者別表示 & 山田 外史, 岩原 正吉 \\
\hline j our nal or & I nt er nat i onal Jour nal of Appl i ed \\
publ i cat i on ti t l e & El ect r onagnet i cs and Mechani cs \\
\hline vol une & 15 \\
\hline nunber & $1-4$ SPEC \\
\hline page range & $15-19$ \\
\hline year & 2001 \\
\hline URL & ht t p: //doi . or g/10. 24517/00049209 \\
\hline
\end{tabular}




\title{
Simulation of amplitude and phase characteristics during inspection of printed circuit board by eddy-current testing probe
}

\author{
Dariusz Kacprzak ${ }^{\mathrm{a}}$, Sotoshi Yamada ${ }^{\mathrm{b}}$ and Masayoshi Iwahara ${ }^{\mathrm{b}}$ \\ ${ }^{a}$ Department of Electrical and Electronic Engineering, School of Engineering, The University of \\ Auckland, Private Bag 92019, Auckland 1, New Zealand \\ Tel.: +649373 7599 extension: 8198; Fax: +649373 746; E-mail: d.kacprzak@auckland.ac.nz \\ ${ }^{\mathrm{b}}$ Faculty of Engineering, Kanazawa University, Kodatsuno 2-40-20, Kanazawa 920-8667, Japan \\ Tel.: +8176234 4942; Fax: +81 79234 4946; E-mail: yamada@magstar.ec.t.kanazawa-u.ac.jp
}

\begin{abstract}
This paper presents simulated amplitude and phase characteristics obtained during the inspection of printed circuit board by an eddy-current testing probe. The simulation is based on the "two-components" nature of the output signal. Amplitude and phase characteristics, obtained during the experimental inspection and simulation, are presented.
\end{abstract}

\section{Introduction}

Eddy current testing (ECT) technique is a very efficient and popular method for the detection of defects and gaps in conductive materials. It has been, applied to the inspection of metal parts, surfaces, pipes and so on. Recently the ECT technique was introduced to the inspection of a printed circuit board (PCB) structure [1]. This new application requires a special sensing device and an adequate method of signal analysis $[2,3]$.

From the whole variety of ECT probes (sensors) a "two coils" probe was selected as the most suitable configuration. One of the coils - an exciting coil, is used to produce a magnetic field, which penetrates PCB's conductors inducing eddy-currents in these conductors. Second coil - a pick-up coil, is used as a sensing device. During the inspection, the probe is scanning the PCB, and the voltage obtained from the pick-up coil is recorded. This voltage signal is a source of information about possible defects. The voltage signal has to be analyzed, so the determination of defects' existence can be provided.

Although there are many methods of signal analysis, all of them are based on the same principle which is detection of peaks/non-linearity on "voltage-versus-sensor position" characteristic. The larger defect is detected, the higher peak on the "voltage-versus-sensor position" characteristic is observed. It is relatively easy to find the relationship between size of the defects/cracks and height of the peak.

Voltage is not the only parameter measured during the inspection. Significant changes between phases of the exciting current and pick-up voltage are also registered [4]. Unlike the "voltage-versus-sensor position", where peaks representing defects differ by amplitudes, the peaks on the "phase-versus-sensor position" characteristic differs also by shapes. In the case of the inspection of PCB, this phenomenon is especially important for determination of signals coming from soldering points. For better interpretation 


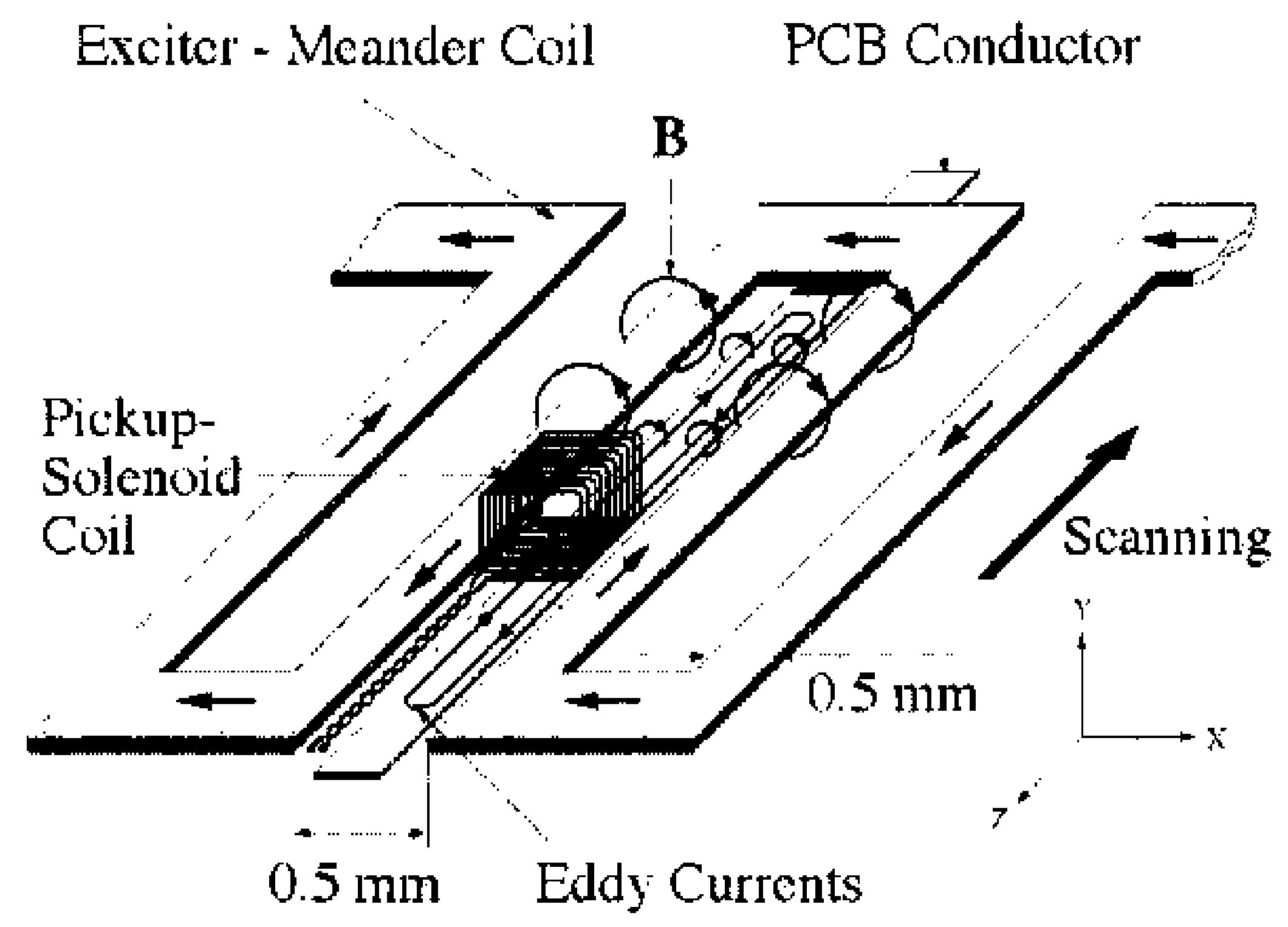

Fig. 1. ECT probe composed of meander - exciting coil and solenoid pick-up coil.

of measured parameters (the voltage and the phase), it is also important to understand roots of shape changes in the "phase-versus-sensor position" (which is explained in this paper).

The paper consisting of three parts firstly provides a brief presentation of the ECT probe. In the second part a theory of the output signal's nature is presented, where sources of changes in the shape of the "phase-versus-sensor position" are explained. The third part includes results from the simulation of amplitude and phase characteristics.

The simulation proves that the changes of the signals' shape/peaks are comprehensible and can be calculated. So the relationship between sizes of defects and signals obtained from inspections can be much more specified. Understanding of voltage and phase changes is a key to determination of the relationship between the "phase-versus-sensor position" and "voltage-versus-sensor position" characteristics. Thus for better analysis of inspected model, both the two characteristic should be taken into account.

\section{Features and construction of the ect probe}

Figure 1 shows a "two-coils configuration" ECT probe used in the inspection of printed circuit boards (PCB). A special designed exciter - the meander coil allows generation of a magnetic field, which causes eddy currents along the PCB conductors. Any defects on the tested conductors appear as obstacles for the eddy currents and change their distributions. The key point of this ECT probe is its pick-up coil, which has a solenoid shape. This kind of pick-up coil allows an inspection of a tangential component of the magnetic flux density B. Because the tangential component appears only in specific cases - together with defects or at conductor's boundaries $[1,2,5]$, the signal delivered by the pick-up coil does not have to be extracted from other signals.

For example: in the case of a defect-less conductor, the tangential component is equal to zero. This is a big advantage of the probe with a solenoid pick-up coil and the main reason for its high sensitivity. During scanning, the probe moves above the test PCB with constant lift-off. The exciting current frequency is $\mathrm{f}=5 \mathrm{MHz}$. Resultant data, obtained from a lock-in amplifier are stored in a PC computer. The Inspection can be performed along or across conductors. However, due to the specific shape of the meander coil and its long conductors, inspection along conductors is preferred.

\section{The nature of the output signal}

The output signal obtained from the ECT probe is composed of two components: 
Component caused by exciting current

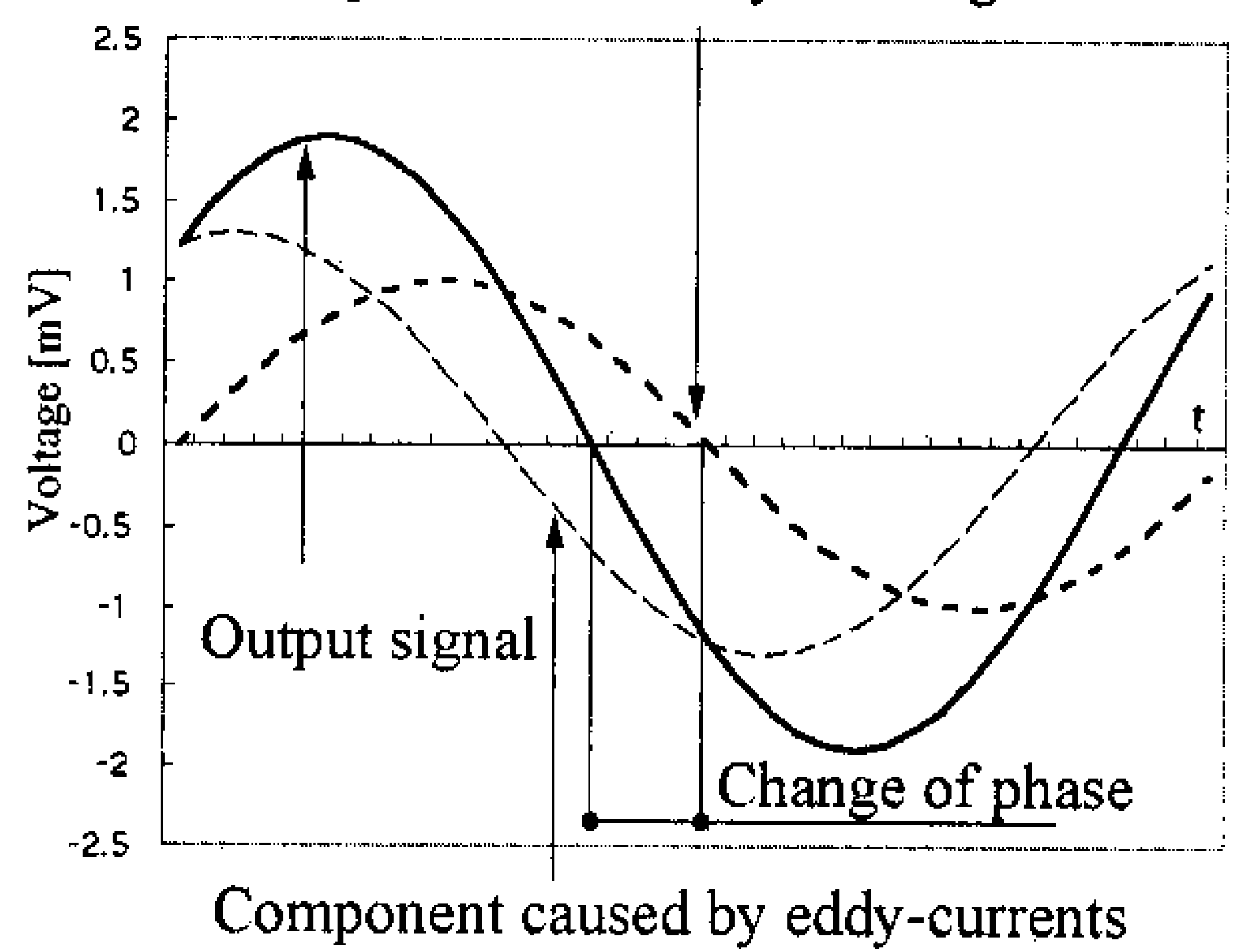

(a)

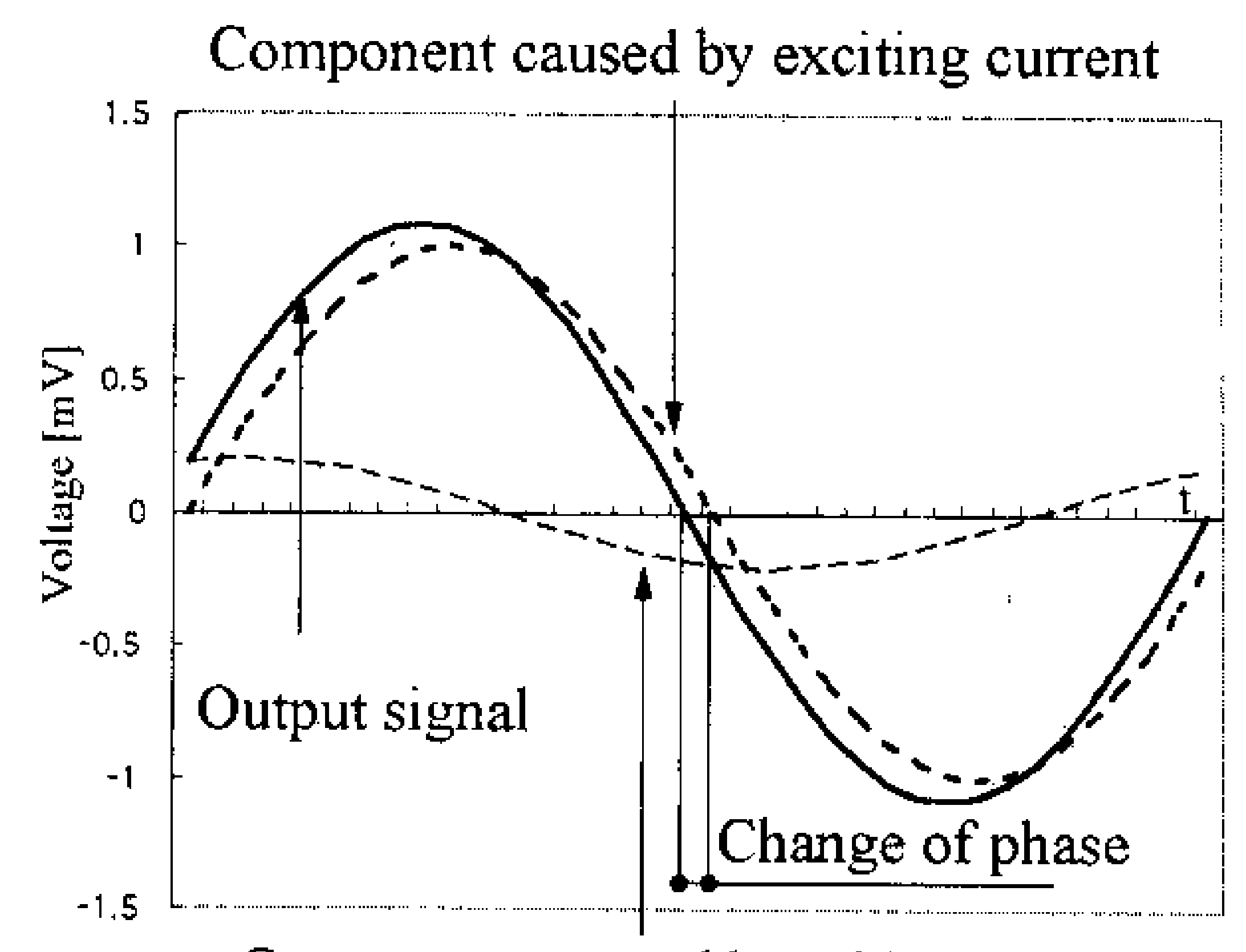

Component caused by eddy-currents

(b)

Fig. 2. Formation of the output signal from two sinusoidal components in cases of (a) high and (b) low component caused by the eddy currents.

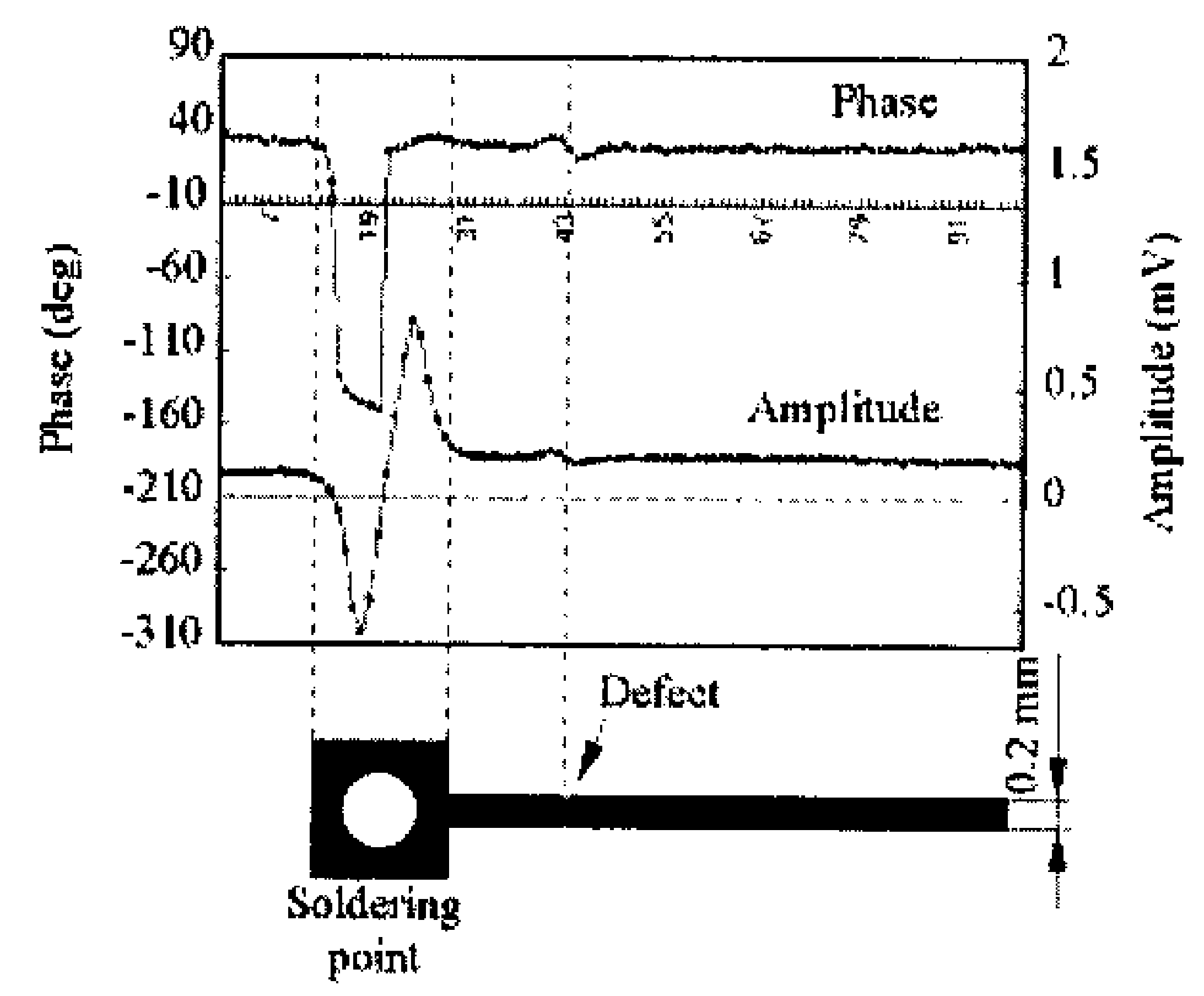

Fig. 3. Amplitude and phase characteristics- experimental results.

- 1 st component caused by an effect of the exciting current on the pick-up coil,

- 2nd component generated directly by the eddy currents.

These two components have the same frequency but different phases, and added together produce at the output signal. The amplitude and the phase of the output signal depend on the proportion of these two components. The first factor affecting this proportion is the position of the pick-up coil. The pick-up coil supposed to be installed parallel to the meander coil conductors, but any deviations from this position are very possible and even unavoidable. The amplitude of the 2 nd component (caused by the eddy-currents) also depends on the width of the inspected element. And it is very large in the case of a soldering point and it is very small in the case of the defect located on a narrow conductor [6].

During the inspection of a homogeneous material, the resultant data obtained from the lock-in amplifier remain constant. In case of the inspection of a PCB conductor with a defect, the amplitude of the 2 nd component increases changing the amplitude and phase of the output signal. Figure 2 presents two 

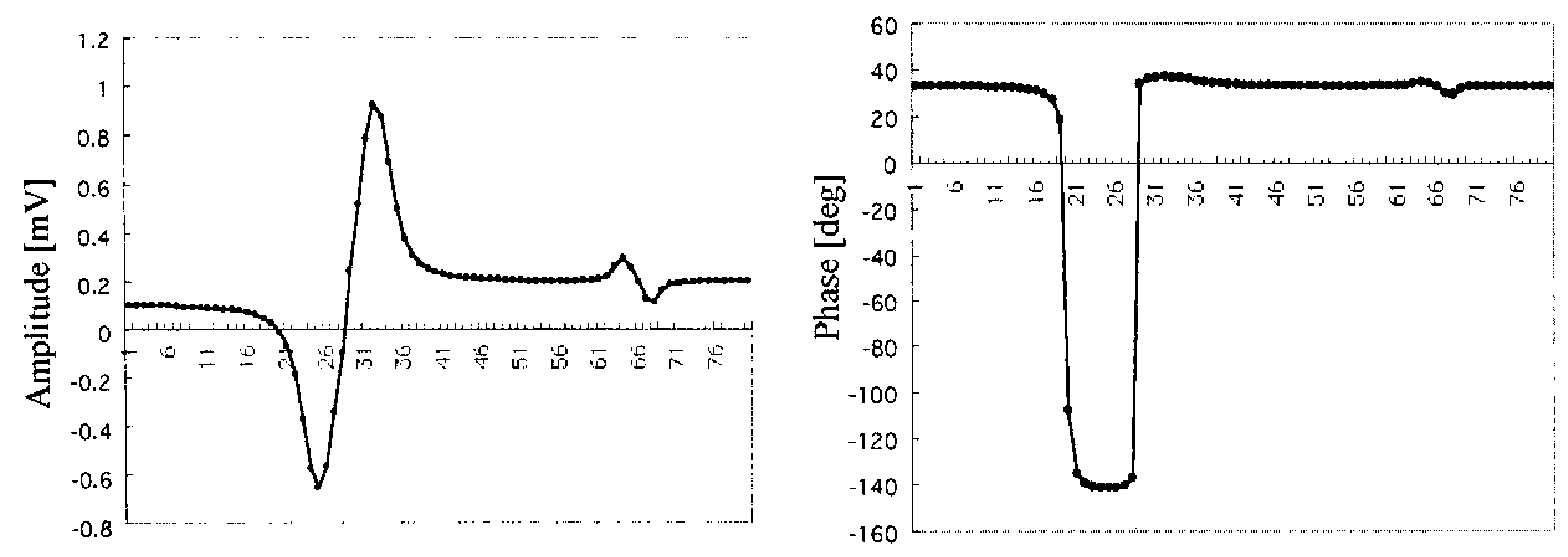

Fig. 4. Amplitude and phase characteristics obtained from the simulation.

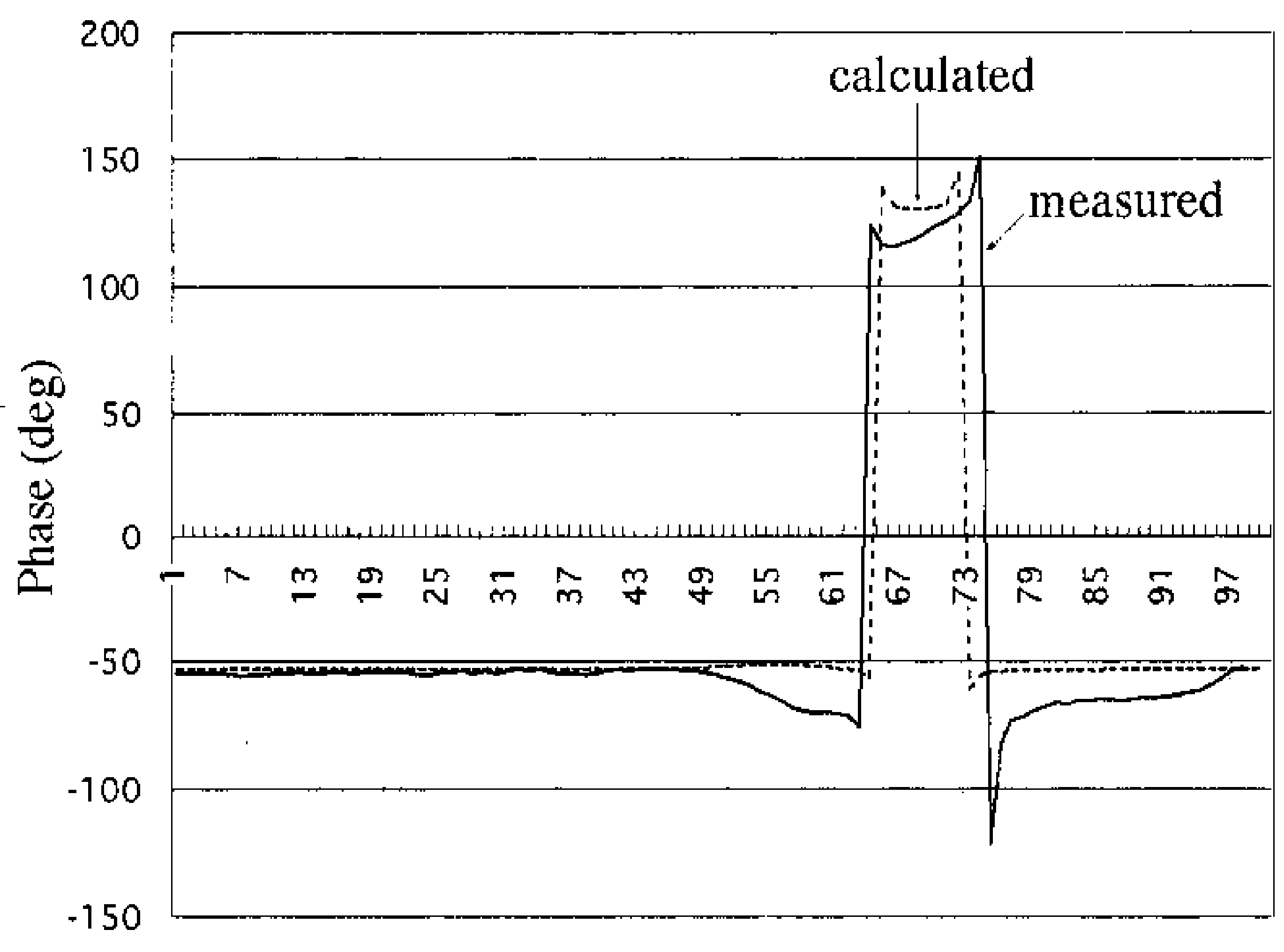

Fig. 5. Phase characteristic obtained from a soldering point - the pick-up coil not parallel to the meander coil's conductors.

characteristics of different proportion between the 1st and 2nd components, which show the changes of the amplitude and the phase.

\section{Simulation of output signal}

A model composed of a conductor and a soldering point was analyzed. Experimental results are presented in Fig. 4. Characteristic "rectangular" changes of the phase exist only during the inspection of soldering points. The "rectangular" shape on the phase characteristic do not appear during the inspection of narrow conductors, where the amplitude of the 2nd component is too small to switch the phase of the total signal and only generates a "wave" form. Based on the diagram showed in Fig. 2 simulations were provided, and the resultant characteristics are presented in Fig. 5.

The characteristic "rectangular" shape representing a soldering point (on the phase characteristic) changes with the position of the pick-up coil. In the case when the pick-up coil is not inserted parallel to the meander coil's conductors, the peak corresponding to the soldering point become deformed as 
shown in Fig. 6. From the analysis phase characteristics much information can be obtained such as: is the pick-up coil is installed parallel to the exciting conductors.

\section{Conclusions}

A study of the structure of the output signal provides an understanding of the signals nature and factors causing changes on the amplitude and the phase characteristics. As the simulation proved, it is possible to predict the shape of amplitude and phase characteristic. Shapes of signals correspond to sizes of defects/obstacles. Characteristic "rectangular" shapes on phase characteristics were obtained only during the inspection of soldering points. Similar shapes can be also obtained during the inspection of a metal surface with rivet holes. However for this inspection the size of the ECT probe has to be adjusted to keep proper proportion of the components.

The "rectangular" shapes on the phase characteristic inspire a new approach to the signal analysis, which is the classification of signals due to their shape.

\section{References}

[1] D. Kacprzak, T. Miyagoshi, S. Yamada and M. Iwahara, Inspection of Printed Circuit Board by ECT Probe with Solenoid Pickup Coil, Journal of J. Magn. Soc. Japan 24(4-2) (2000), 839-842.

[2] D. Kacprzak, S. Yamada and M. Iwahara, Analysis of the structure of printed circuit boards by means of the eddy current technique, INSIGHT Non-Destructive testing and Condition Monitoring (The Journal of The British Institute of Non-Destructive Testing) 42(5) (May 2000).

[3] T. Taniguchi, D. Kacprzak, S. Yamada, M. Iwahara and T. Miyagoshi, Defect Detection of Printed Circuit Board by Using Eddy Current Testing Technique and Image Processing, in: Electromagnetic Non-destructive Evaluation, (Vol. IV), S.S. Udpa et al., eds., IOS Press, 2000, pp. 111-119.

[4] H. Tsuboi, M. Tanaka, M. Kimura AND K. Ikeda, Three-dimensional finite element analysis of nondestructive testing of metallic tubular product, IEEE Transactions on Magnetics 35(5:2) (Sept. 1999), 3721-3723.

[5] D. Kacprzak, T. Miyagoshi, T. Tanigushi, S. Yamada and M. Iwahara, Comparison of two types of pick-up coil for meander excitation, in: Non-linear Electromagnetic Systems, P.Di Barba and A. Savini, eds, IOS Press, 2000, pp. $229-232$.

[6] D. Kacprzak, T. Taniguchi, S. Yamada, M. Iwahara and T. Miyagoshi, Interpretation of Printed Circuit Boards Structure via Amplitude and Signal Phase Obtained from ECT Testing, 6th Int. Workshop on Electromagnetic Non-destructive Evaluation, 2000.6. 